\title{
Modulational instability and nonlocality management in coupled NLS system
}

\author{
Evgeny V Doktorov and Maxim A Molchan \\ B I Stepanov Institute of Physics, 220072 Minsk, Belarus \\ E-mail: doktorov@dragon.bas-net.by, m.moltschan@dragon. bas-net.by
}

\begin{abstract}
The modulational instability of two interacting waves in a nonlocal Kerrtype medium is considered analytically and numerically. For a generic choice of wave amplitudes, we give a complete description of stable/unstable regimes for zero groupvelocity mismatch. It is shown that nonlocality suppresses considerably the growth rate and bandwidth of instability. For nonzero group-velocity mismatch we perform a geometrical analysis of a nonlocality management which can provide stability of waves otherwise unstable in a local medium.

PACS numbers: 42.65.Sf, 42.65.Jx, 52.35.Mw
\end{abstract}

Submitted to: Physica Scripta 


\section{Introduction}

Various nonlinear dispersive wave systems exhibit instability, known as the modulational instability (MI). MI stems from the interaction between nonlinearity and group-velocity dispersion and manifests itself as a self-induced amplitude modulation of a continuous wave propagating in a nonlinear medium, with subsequent generation of localized structures. MI arises in many physical settings including plasma [1, 2, 3, 4], fluids [5], nonlinear optics [6, 7, 8, 9], and atomic Bose-Einstein condensates [10, 11].

In the context of nonlinear fibre optics, MI requires anomalous dispersion of a medium to exist. In this case, negative group-velocity dispersion combined with the self-phase modulation amplifies the modulational frequency sidebands producing a train of ultra-short pulses as a result of breakup of a continuous wave [12, 8, 13].

On the other hand, when two or more optical beams propagate down the fibre, MI can occur even in the normal-dispersion regime, at the cost of the cross-phase modulation [14, 15]. Such a situation is usually described by a set of coupled nonlinear Schrödinger (NLS) equations. A thorough analysis of MI for two nonlinearly interacting waves in a Kerr medium was performed in recent papers [16, 17].

The above results concern the class of local nonlinear media when the response of the medium at a particular point depends solely on the wave intensity at that point. Alternatively, nonlocality plays a key role in physical systems where transport phenomena and finite-range interaction cannot be neglected. The examples of importance of spatial nonlocality for the development of MI can be found in plasmas [18, 19], liquid crystals [20], discrete nonlinear systems [21], Bose-Einstein condensates [22]. In particular, nonlocality changes drastically the growth rate and bandwidth of instability caused by stochasticity of parameters of a Kerr medium [23]. The present status of MI of scalar waves in nonlocal media is discussed in the review papers [24, 25].

In our paper we extend the analysis of MI in coupled wave systems to the case of nonlocal media. It was demonstrated recently that vector soliton structures in nonlocal media exhibit properties that have no counterpart in the scalar case [26]. In particular, a possibility was revealed to stabilize multipole (dipole, quadrupole, hexapole, etc.) vector solitons in bulk nonlocal media at the cost of vectorial coupling [27]. In this case mutually incoherent nodeless and multipole components jointly induce a nonlinear refractive index profile. As regards higher-order nonlinearities, a one-dimensional phenomenological model of a nonlocal medium with focusing cubic and defocusing quintic nonlinearities was developed in [28]. Two types of solitons, even-parity (fundamental) and odd-parity (dipole) were found and their stability was explored. Note that solitons of this sort are unstable in local media. Lastly, an interesting result should be mentioned concerning formation of stable three-dimensional spatiotemporal solitons in nonlocal Kerr media [29]. Fundamental soliton of this model is stable provided its propagation constant exceeds a certain critical value inverse proportional to a nonlocality parameter. 
Below we derive the dispersion relation which characterizes the propagation of two interacting waves in a nonlocal Kerr-type medium. For the case of zero group-velocity mismatch we are able to perform a complete analytic description of stability/instability regimes for generic wave amplitudes. It is shown that nonlocality suppresses considerably the growth rate and bandwidth of instability. For nonzero velocity mismatch, we investigate a role of nonlocality in a management of the stability properties in the system under consideration. Nonlocality is shown to provide an efficient stabilization mechanism permitting stabilize interacting waves which are strongly unstable in a local medium.

\section{Model}

Propagation of two nonlinearly interacting waves with complex amplitudes $u(t, x)$ and $v(t, x)$ in a nonlocal Kerr medium is modelled by the following system of dimensionless equations:

$$
\begin{aligned}
\mathrm{i}\left(\frac{\partial u}{\partial t}+V_{1} \frac{\partial u}{\partial x}\right) & +\frac{d_{1}}{2} \frac{\partial^{2} u}{\partial x^{2}} \\
& +s_{1} u \int \mathrm{d} x^{\prime} R\left(x-x^{\prime}\right)\left(\left|u\left(t, x^{\prime}\right)\right|^{2}+\alpha\left|v\left(t, x^{\prime}\right)\right|^{2}\right)=0 \\
\mathrm{i}\left(\frac{\partial v}{\partial t}+V_{2} \frac{\partial v}{\partial x}\right) & +\frac{d_{2}}{2} \frac{\partial^{2} v}{\partial x^{2}} \\
& +s_{2} v \int \mathrm{d} x^{\prime} R\left(x-x^{\prime}\right)\left(\left|v\left(t, x^{\prime}\right)\right|^{2}+\alpha\left|u\left(t, x^{\prime}\right)\right|^{2}\right)=0
\end{aligned}
$$

which naturally generalize those [30] for a local medium.

Physical meaning of coordinates $t$ and $x$ depends on the context of the problem: they are time and coordinate for Bose-Einstein condensates, longitudinal coordinate $z$ and transversal coordinate $x$ for optical beams in waveguides, the propagation coordinate $z$ and time $t$ for optical pulses in fibres, respectively. The parameters $d_{j}$ $(j=1,2)$ determine effective atom masses in condensate models, diffraction coefficients in waveguides and group-velocity dispersion for pulses. The parameters $V_{j}$ stand for group velocities, $s_{j}$ determine nonlinearity coefficients, and $\alpha>0$ is a cross-phase modulation parameter. No restrictions are posed on signs and magnitudes of $d_{j}$ and $s_{j}$. $R(x)$ is a positively definite symmetric response function of a nonlinear medium obeying the normalization condition $\int \mathrm{d} x R(x)=1$ (we do not specify the integration limits

when the integration is carried out along the whole line). An important development of nonlocal models with asymmetric response functions relevant, e.g., to account for the Raman effect, was achieved by Wyller [31].

We will illustrate our results by the exponential response function

$$
R(x)=\frac{1}{2 \sigma} \exp \left(-\frac{|x|}{\sigma}\right) .
$$

Here $\sigma$ is a nonlocality parameter. The function (2) occurs as a solution of the diffusiontype equation for nonlinearity [26] and adequately describes the nonlinear response of 
thermo-optical materials, liquid crystals and partially ionized plasmas. For $\sigma \rightarrow 0$ we have $R(x) \rightarrow \delta(x)$ and hence reproduce the local model used in [17].

\section{Modulational instability: general analysis}

In order to investigate the modulational instability in the system (1), we start with the continuous wave solution of the form

$$
u=u_{0} \mathrm{e}^{-\mathrm{i} \omega_{1} t}, \quad v=v_{0} \mathrm{e}^{-\mathrm{i} \omega_{2} t},
$$

where $u_{0}$ and $v_{0}$ are constant real amplitudes, and frequencies $\omega_{j}$ are given by the relations

$$
\omega_{1}=-s_{1}\left(u_{0}^{2}+\alpha v_{0}^{2}\right), \quad \omega_{2}=-s_{2}\left(\alpha u_{0}^{2}+v_{0}^{2}\right),
$$

which are the same as for the local coupled NLS system.

Following the standard procedure, let us consider a small perturbation of the continuous waves (3):

$$
u=\left(u_{0}+a(t, x)\right) \mathrm{e}^{-\mathrm{i} \omega_{1} t}, \quad v=\left(v_{0}+b(t, x)\right) \mathrm{e}^{-\mathrm{i} \omega_{2} t} .
$$

Substituting (41) into (11) and neglecting terms with the second and higher orders of the complex perturbation amplitudes $a$ and $b$, we obtain a system of linear equations

$$
\begin{gathered}
\mathrm{i}\left(\frac{\partial a}{\partial t}+V_{1} \frac{\partial a}{\partial x}\right)+\frac{d_{1}}{2} \frac{\partial^{2} a}{\partial x^{2}}+s_{1} u_{0}^{2} \int \mathrm{d} x^{\prime} R\left(x-x^{\prime}\right)\left(a+a^{*}\right)\left(t, x^{\prime}\right) \\
+\alpha s_{1} u_{0} v_{0} \int \mathrm{d} x^{\prime} R\left(x-x^{\prime}\right)\left(b+b^{*}\right)\left(t, x^{\prime}\right)=0, \\
\mathrm{i}\left(\frac{\partial b}{\partial t}+V_{2} \frac{\partial b}{\partial x}\right)+\frac{d_{2}}{2} \frac{\partial^{2} b}{\partial x^{2}}+s_{2} v_{0}^{2} \int \mathrm{d} x^{\prime} R\left(x-x^{\prime}\right)\left(b+b^{*}\right)\left(t, x^{\prime}\right) \\
+\alpha s_{2} u_{0} v_{0} \int \mathrm{d} x^{\prime} R\left(x-x^{\prime}\right)\left(a+a^{*}\right)\left(t, x^{\prime}\right)=0 .
\end{gathered}
$$

We seek for solutions of (15) in the form

$$
\begin{aligned}
& a=A_{+} \mathrm{e}^{\mathrm{i}(\kappa x-\Omega t)}+A_{-}^{*} \mathrm{e}^{-\mathrm{i}\left(\kappa x-\Omega^{*} t\right)}, \\
& b=B_{+} \mathrm{e}^{\mathrm{i}(\kappa x-\Omega t)}+B_{-}^{*} \mathrm{e}^{-\mathrm{i}\left(\kappa x-\Omega^{*} t\right)},
\end{aligned}
$$

where $\kappa$ and $\Omega$ are the wave number and frequency of the perturbation, respectively. Hence, the condition of solvability of the system (5) takes the form of the dispersion relation

$$
\left[\left(\Omega-V_{1} \kappa\right)^{2}-\Omega_{1}^{2}\right]\left[\left(\Omega-V_{2} \kappa\right)^{2}-\Omega_{2}^{2}\right]-\Omega_{12}^{4}=0
$$

where

$$
\begin{gathered}
\Omega_{1}^{2}=\frac{1}{4} d_{1} \kappa^{2}\left(d_{1} \kappa^{2}-4 s_{1} u_{0}^{2} \hat{R}\right), \quad \Omega_{2}^{2}=\frac{1}{4} d_{2} \kappa^{2}\left(d_{2} \kappa^{2}-4 s_{2} v_{0}^{2} \hat{R}\right) \\
\Omega_{12}^{4}=d_{1} d_{2} s_{1} s_{2}\left(\alpha u_{0} v_{0} \kappa^{2} \hat{R}\right)^{2} .
\end{gathered}
$$

Note the appearance of the Fourier transform $\hat{R}(\kappa)$ of the response function $R(x)$,

$$
\hat{R}(\kappa)=\int \mathrm{d} x R(x) \mathrm{e}^{\mathrm{i} \kappa x}=\frac{1}{1+\sigma^{2} \kappa^{2}}, \quad \hat{R}(-\kappa)=\hat{R}^{*}(\kappa),
$$


in the expressions for $\Omega_{j}^{2}$ and $\Omega_{12}^{4}$. Since $\hat{R}(\kappa) \rightarrow 1$ for $\sigma \rightarrow 0$, the expression (6) reduces in this limit to the well-known dispersion relation for the local model [15, 16, 17].

The dispersion relation (6) takes the form of a general fourth-order algebraic equation for $\Omega$. In order to provide better insight into the properties of its solutions, we begin the analysis from the particular case of equal group velocities, $V_{1}=V_{2}$, which allows us to perform the complete analytical treatment.

\section{Equal group velocities}

If $V_{1}=V_{2} \equiv V$, we can eliminate the terms with $V_{j}$ from (5) by the Galilean transformation $t^{\prime}=t, x^{\prime}=x-V t$, and the dispersion relation (6) reduces to the biquadratic polynomial in $\Omega$,

$$
\left(\Omega^{2}-\Omega_{1}^{2}\right)\left(\Omega^{2}-\Omega_{2}^{2}\right)-\Omega_{12}^{4}=0 .
$$

Introducing the definitions

$$
\begin{aligned}
& \mu_{j}=s_{j} d_{j}, \quad \varphi^{2}=\frac{1}{4}\left(d_{1}^{2}+d_{2}^{2}\right), \\
& \psi=\mu_{1} u_{0}^{2}+\mu_{2} v_{0}^{2}, \quad N(\kappa)=\kappa^{-2} \hat{R}(\kappa),
\end{aligned}
$$

we write equation (9) in the form

$$
\begin{aligned}
& \Omega^{4}-\kappa^{4}\left(\varphi^{2}-N \psi\right) \Omega^{2}+ \\
& \quad \frac{\kappa^{2}}{16}\left[d_{1}^{2} d_{2}^{2}-4 N\left(\mu_{1} d_{2}^{2} u_{0}^{2}+\mu_{2} d_{1}^{2} v_{0}^{2}\right)+16 N^{2}\left(1-\alpha^{2}\right) \mu_{1} \mu_{2} u_{0}^{2} v_{0}^{2}\right]=0 .
\end{aligned}
$$

In the local models, stability properties are determined directly by the analysis of the function $\Omega(\kappa)$. In contrast, to establish general properties of the stability/instability domains for the nonlocal case, we will primarily consider the function $\Omega(N)$. More detailed information will be then provided by a particular choice of the positively definite function $N(\kappa)$.

Solving (11) gives

$$
\Omega_{ \pm}^{2}=\frac{1}{2} \kappa^{4}\left(\varphi^{2}-N \psi \pm \sqrt{D}\right)
$$

where

$$
D(N)=4 \Delta_{1} N^{2}+4 \delta \eta N+\delta^{2}
$$

and

$$
\begin{gathered}
\Delta_{1}=\mu_{1} \mu_{2}\left(\alpha u_{0} v_{0}\right)^{2}+\eta^{2}, \quad \eta=\frac{1}{2}\left(\mu_{1} u_{0}^{2}-\mu_{2} v_{0}^{2}\right), \\
\delta=\frac{1}{4}\left(d_{1}^{2}-d_{2}^{2}\right) .
\end{gathered}
$$

Without loss of generality we take $\delta \geq 0$.

Stability of continuous waves (3) will be ensured if both $\Omega_{+}^{2}$ and $\Omega_{-}^{2}$ are positive for any choice of the continuous wave amplitudes $u_{0}$ and $v_{0}$. In other words, we do not consider situations when stability/instability conditions fulfil only for specific relations between $u_{0}$ and $v_{0}$, because they are not generally satisfied. Evidently, we get $\Omega_{ \pm}^{2}>0$ if $D>0$ and $\sqrt{D}<\varphi^{2}-N \psi$. 


\subsection{Analysis}

To reveal positiveness regions of $\Omega_{ \pm}^{2}$, we analyze at first the function $D(N)$ (13). Positiveness of $D(N)$ depends on $\Delta_{1}$ and $\eta$. It is seen from (14) that $\Delta_{1}>0$ for any $\alpha, u_{0}$ and $v_{0}$ if $\mu_{1} \mu_{2}>0$, as well as for $\alpha<1$ if $\mu_{1} \mu_{2}<0$. Note that $\Delta_{1}$ could be positive for $\mu_{1} \mu_{2}<0$ and $\alpha>1$ under a specific choice of $u_{0}$ and $v_{0}$, but we disregard such a possibility, as was stated above. Hence, in what follows we consider $\Delta_{1}$ to be positive for generic choice of $u_{0}$ and $v_{0}$, and for only restriction $\alpha<1$ if $\mu_{1} \mu_{2}<0$.

As regards the role of $\eta$, it can be elucidated from the analysis of the discriminant $D_{1}=-16 \mu_{1} \mu_{2}\left(\alpha \delta u_{0} v_{0}\right)^{2}$ of the equation $D(N)=0$. Evidently, $D>0$ if $D_{1}<0$, i.e., for $\mu_{1} \mu_{2}>0$ and arbitrary $\alpha$ (the parabola $D(N)=0$ lies above the horizontal axis $N$ ). If $D_{1}>0$ that occurs for $\mu_{1} \mu_{2}<0$ and hence for $\alpha<1$ (two real intersection points of the parabola with the $N$ axis), we have two possibilities for $D>0$, depending on the sign of $\eta$ :

(i) $\eta>0$ means $\mu_{1}<0$ and $\mu_{2}<0$. Both intersection points lie on the negative (unphysical) part of the $N$ axis, and $D>0$ for any $N>0$;

(ii) $\eta<0$ means $\mu_{1}<0$ and $\mu_{2}>0$. Hence, $D>0$ for $N \in\left(0, N_{-}^{\prime}\right) \cup\left(N_{+}^{\prime}, \infty\right)$,

where

$$
N_{ \pm}^{\prime}=\frac{\delta}{2 \Delta_{1}}\left( \pm \sqrt{\eta^{2}-\Delta_{1}}+|\eta|\right)>0
$$

Lastly, $D<0$ if $D_{1}>0, \alpha<1, \eta<0$, and $N \in\left(N_{-}^{\prime}, N_{+}^{\prime}\right)$. Let us summarize the information obtained from the above analysis for the generic $u_{0}$ and $v_{0}$ :

$$
D>0 \text { for } \Delta_{1}>0 \text { and }
$$

(i) $\mu_{1} \mu_{2}>0, \forall(\alpha, N)$;

(ii) $\mu_{1}>0, \mu_{2}<0, \alpha<1, \forall N$;

(iii) $\mu_{1}<0, \mu_{2}>0, \alpha<1, N \in\left(0, N_{-}^{\prime}\right) \cup\left(N_{+}^{\prime}, \infty\right)$;

$$
D<0 \text { for } \Delta_{1}>0 \text { and }
$$

(iv) $\mu_{1}<0, \mu_{2}>0, \alpha<1, N \in\left(N_{-}^{\prime}, N_{+}^{\prime}\right)$.

To study the condition $\sqrt{D}<\varphi^{2}-N \psi$, we introduce $Z=D-\left(\varphi^{2}-N \psi\right)^{2}$, or

$$
Z=4 \Delta_{2} N^{2}+4 f N+g
$$

where

$$
\begin{aligned}
& \Delta_{2}=\left(\alpha^{2}-1\right) \mu_{1} \mu_{2} u_{0}^{2} \mu_{2}^{2}, \quad f=\frac{1}{4}\left(\mu_{1} d_{1}^{2} u_{0}^{2}+\mu_{2} d_{2}^{2} v_{0}^{2}\right), \\
& g=\frac{1}{4} d_{1}^{2} d_{2}^{2} .
\end{aligned}
$$

They are negative values of $Z$ that correspond to the condition $\sqrt{D}<\varphi^{2}-N \psi$, and we require $\varphi^{2}>N \psi$. 
The discriminant $D_{2}=16\left(f^{2}-\Delta_{2} g\right)$ of the equation $Z(N)=0$ has the form

$$
D_{2}=\left(\mu_{1} d_{1}^{2} u_{0}^{2}+\mu_{2} d_{2}^{2} v_{0}^{2}\right)^{2}-4\left(1-\alpha^{2}\right) \mu_{1} \mu_{2}\left(d_{1} d_{2} u_{0} v_{0}\right)^{2} .
$$

It is seen that $D_{2}<0$ occurs only for special requirements on $u_{0}$ and $v_{0}$; hence, we take in what follows $D_{2}>0$ for generic $u_{0}$ and $v_{0}$. This condition takes place for $\mu_{1} \mu_{2}>0$ and any $\alpha$, as well as for $\mu_{1} \mu_{2}<0$ and $\alpha<1$.

Now we can establish properties of $Z(N)$. First of all we see that the presence of $f$ in (16) excludes the case $\mu_{1} \mu_{2}<0$ because it would lead to nongeneric conditions on $u_{0}$ and $v_{0}$. Solutions of $Z=0$ are written as

$$
Z_{ \pm}=\frac{1}{2 \Delta_{2}}\left( \pm \sqrt{f^{2}+\frac{1}{4} d_{1}^{2} d_{2}^{2} \Delta_{2}}-f\right) .
$$

Since $D_{2}>0$, both $Z_{+}$and $Z_{-}$are real. At first we consider the case $\Delta_{2}>0$ which occurs for $\mu_{1} \mu_{2}>0$ and $\alpha>1$. If $f>0$, i.e. $\mu_{1}>0, \mu_{2}>0$, and hence $\alpha>1$, we obtain $Z_{+}>0$ and $Z_{-}<0$. Therefore, $Z$ will be positive for $N>Z_{+}$and negative for $N \in\left(0, Z_{+}\right)$. For $f<0$, i.e., $\mu_{1}<0, \mu_{2}<0$ and $\alpha>1$, the solutions of $Z(N)=0$,

$$
Z_{ \pm}^{\prime}=\frac{1}{2 \Delta_{2}}\left( \pm \sqrt{f^{2}+\frac{1}{4} d_{1}^{2} d_{2}^{2} \Delta_{2}}+|f|\right)
$$

obey the same properties as $Z_{ \pm}$, i.e., $Z>0$ for $N>Z_{+}^{\prime}$ and $Z<0$ for $N \in\left(0, Z_{+}^{\prime}\right)$.

If $\Delta_{2}<0$ (i.e., $\mu_{1} \mu_{2}>0$ and $\alpha<1$ ), both roots of $Z(N)=0$ are positive for $f>0$ $\left(\mu_{1}>0, \mu_{2}>0\right)$

$$
\tilde{Z}_{ \pm}=\frac{1}{4\left|\Delta_{2}\right|}\left(d_{1}^{2} u_{0}^{2}+d_{2}^{2} v_{0}^{2} \pm \sqrt{\left(d_{1}^{2} u_{0}^{2}+d_{2}^{2} v_{0}^{2}\right)^{2}-\left|\Delta_{2}\right| d_{1}^{2} d_{2}^{2}}\right)>0,
$$

and negative for $f<0\left(\mu_{1}<0\right.$ and $\left.\mu_{2}<0\right)$,

$$
\tilde{Z_{ \pm}^{\prime}}=-\frac{1}{4\left|\Delta_{2}\right|}\left(d_{1}^{2} u_{0}^{2}+d_{2}^{2} v_{0}^{2} \pm \sqrt{\left(d_{1}^{2} u_{0}^{2}+d_{2}^{2} v_{0}^{2}\right)^{2}-\left|\Delta_{2}\right| d_{1}^{2} d_{2}^{2}}\right)<0 .
$$

Hence, for $\Delta_{2}<0$ we have $Z(N)>0$ for $f>0$ and $N \in\left(\tilde{Z}_{-}, \tilde{Z}_{+}\right)$, while $Z<0$ for $f>0$ and $N \in\left(0, \tilde{Z}_{-}\right) \cup\left(\tilde{Z}_{+}, \infty\right)$, as well as for $f<0$ and any $N$.

In addition, we should provide the condition $\varphi^{2}>N \psi$ to fulfil. Two possibilities exist to satisfy this condition, depending on the signs of $\mu_{j}$ :

(i) for $\mu_{1}>0$ and $\mu_{2}>0$ and any $\alpha$ we should assume the restriction $N<B$ to fulfil, where

$$
B=\frac{1}{4} \frac{d_{1}^{2}+d_{2}^{2}}{\mu_{1} u_{0}^{2}+\mu_{2} v_{0}^{2}}
$$

(ii) there are no restrictions on $N$ when $\mu_{1}<0$ and $\mu_{2}<0$ for generic $u_{0}$ and $v_{0}$ and any $\alpha$.

On the other hand, $\varphi^{2}<N \psi$ for $\mu_{1}>0, \mu_{2}>0$ and $N>B$. 


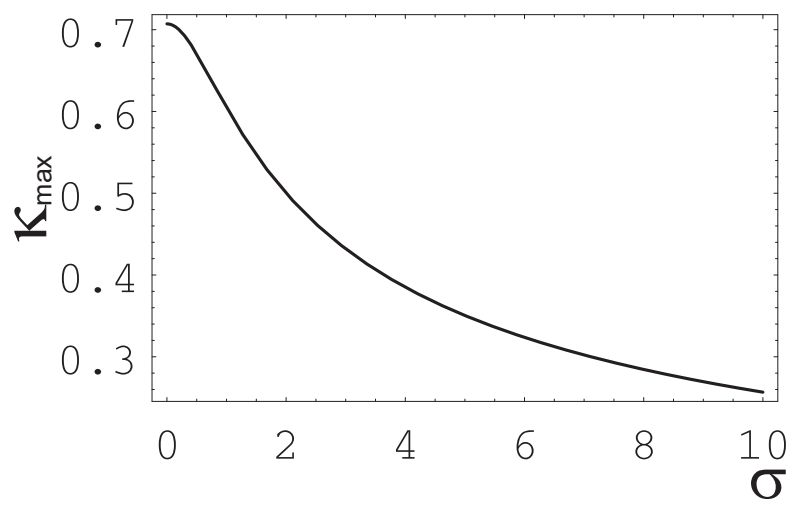

Figure 1. Maximum wave number $\kappa_{\max }$ of the instability range $\left(0, \kappa_{\max }\right)$ versus the nonlocality parameter $\sigma$. Nonlocality suppresses the instability bandwidth. The instability regime corresponds to the case (g) in (20).

\subsection{Summary of the analysis}

Summarizing the results obtained from the analysis of solutions $\Omega_{ \pm}^{2}(12)$, we infer that stability regions $\left(D>0, \sqrt{D}<\varphi^{2}-N \Psi\right)$ are determined by the following conditions on the parameters of equations (1) for generic choice of the amplitudes $u_{0}$ and $v_{0}$ :

(a) $\mu_{1}<0, \mu_{2}<0, \alpha<1, \forall N$;

(b) $\mu_{1}>0, \mu_{2}>0, \alpha>1, N \in\left(0, \min \left(B, Z_{+}\right)\right)$;

(c) $\mu_{1}<0, \mu_{2}<0, \alpha>1, N \in\left(0, Z_{+}^{\prime}\right)$;

(d) $\mu_{1}>0, \mu_{2}>0, \alpha<1,\left\{\begin{array}{cc}N \in\left(0, \min \left(B, \tilde{Z_{-}}\right)\right), & B<\tilde{Z_{+}}, \\ N \in\left(0, \tilde{Z_{-}}\right) \cup\left(\tilde{Z_{+}}, B\right), & \text { if } \tilde{Z_{+}}<B .\end{array}\right.$

Accordingly, instability regions are characterized by the parameters

(e) $\mu_{1}<0, \mu_{2}>0, \alpha<1, N \in\left(N_{-}^{\prime}, N_{+}^{\prime}\right)$;

(f) $\mu_{1}>0, \mu_{2}>0, \alpha>1, N>\min \left(B, Z_{+}\right)$;

(g) $\mu_{1}<0, \mu_{2}<0, \alpha>1, N>Z_{+}^{\prime}$;

(h) $\mu_{1}>0, \mu_{2}>0, \alpha<1, \begin{cases}N \in\left(\tilde{Z}_{-}, \tilde{Z}_{+}\right), & \text {if } B<\tilde{Z}_{-}, \\ N \in\left(B, \tilde{Z}_{+}\right), & \text {if } B \in\left(\tilde{Z}_{-}, \tilde{Z}_{+}\right), \\ N>B, & \text { if } B>\tilde{Z}_{+} .\end{cases}$

It should be noted that stability properties depend on the sign of the products $\mu_{j}=s_{j} d_{j}$ and not on $s_{j}$ and $d_{j}$ separately. Note also that we reproduce the results of Kourakis and Shukla [17] in the local limit $\left(N=\kappa^{-2}\right)$. At the same time, the availability of an additional degree of freedom in the form of nonlocality enables us to manage the conditions of stability/instability. In the next subsection we demonstrate this possibility. 


\subsection{Examples}

The instability growth rate is characterized by the quantity

$$
\lambda(\kappa)=\max \left(\left|\operatorname{Im}\left(\sqrt{\Omega_{-}^{2}}\right)\right|,\left|\operatorname{Im}\left(\sqrt{\Omega_{+}^{2}}\right)\right|\right) .
$$

Below we use the exponential response function (2). For this function we can invert the dependence $N(\kappa)$ :

$$
\kappa^{2}=\frac{1}{2 \sigma^{2}}\left(\sqrt{1+\frac{4 \sigma^{2}}{N}}-1\right)
$$

We fix magnitudes of all the parameters, except for $\sigma$, to reveal the role of nonlocality in the development of MI for a number of regimes listed in (20). As regards the crossphase modulation parameter $\alpha$, we take its featured values, namely, $\alpha=2$ for $\alpha>1$ and $\alpha=2 / 3$ for $\alpha<1$. At first we consider the regime (g) in (20) and choose the parameters as $\mu_{1}=\mu_{2}=-1, u_{0}=v_{0}=1, d_{1}^{2}=d_{2}^{2}=4, \alpha=2$. They correspond to the defocusing nonlinearity in planar waveguides or normal dispersion in a focusing Kerr medium (recall that MI does not occur in such conditions for a single field). Since $N>Z_{+}^{\prime}$ for the regime $(\mathrm{g})$, we have $\kappa \in\left(0, \kappa_{\max }\right)$ from (17) with

$$
\kappa_{\max }^{2}=\frac{1}{2 \sigma^{2}}\left(\sqrt{1+\frac{4 \sigma^{2}}{Z_{+}^{\prime}}}-1\right) .
$$

Figure 11 shows the dependence $\kappa_{\max }(\sigma)$ with $Z_{+}^{\prime}$ from (17). We see that the range $\left(0, \kappa_{\max }\right)$ of instability wave numbers narrows with growing nonlocality. Figure 2 shows the MI gain spectra $\lambda(\kappa)$ (21) for three values of the nonlocality parameter $\sigma$. The maximum gain $\lambda_{\max }$ decreases with an increase in the nonlocality parameter $\sigma$. Such a decrease is illustrated by figure 3 .

The second example covers the regime (e) in (20). This regime describes a joint propagation of two waves, when one of them 'sees' the normal dispersion of a medium, while the second wave moves in the anomalous dispersion environment. Since the allowable values of $N$ lie within the interval $\left(N_{-}^{\prime}, N_{+}^{\prime}\right)$, we have two limiting values of the modulation wave numbers, namely, $\kappa_{-}$and $\kappa_{+}$:

$$
\kappa_{ \pm}=\frac{1}{2 \sigma^{2}}\left(\sqrt{1+\frac{4 \sigma^{2}}{N_{\mp}^{\prime}}}-1\right)
$$

We choose $\mu_{1}=-1, \mu_{2}=1, u_{0}=v_{0}=1, d_{1}^{2}=2, d_{2}^{2}=4, \alpha=2 / 3$. As it is seen from figure 4, the interval width $\left(\kappa_{-}, \kappa_{+}\right)$decreases with increasing $\sigma$. Owing to the dependence of $\kappa_{ \pm}$on the nonlocality parameter $\sigma$, the MI gain spectra demonstrate different boundary values of the bandwidth position, both minimal and maximal, for different $\sigma$ (figure 5 ).

\section{Group-velocity mismatch and nonlocality management}

When we account for group-velocity mismatch $\left(V_{1} \neq V_{2}\right)$, the dispersion relation (6) gives a general fourth-order algebraic equation. To avoid cumbersome calculation, we 


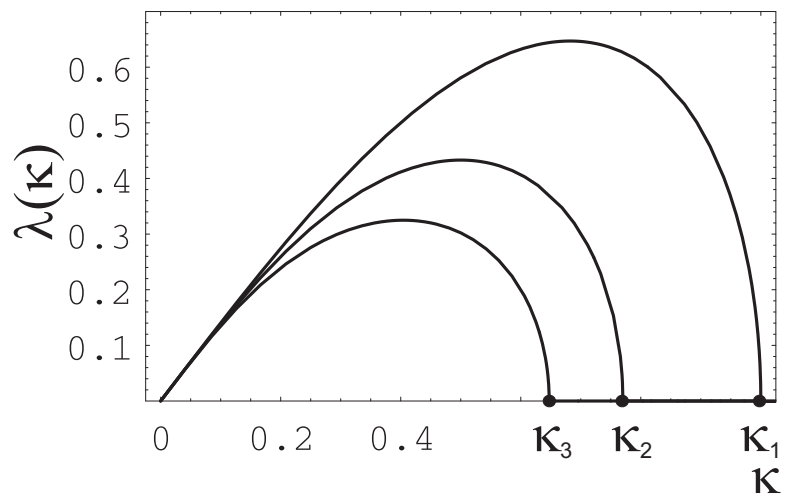

Figure 2. MI gain spectra for the instability regime (g). The points $\kappa_{1}, \kappa_{2}$ and $\kappa_{3}$ corresponds to $\kappa_{\max }$ in figure 1 for $\sigma=1,2$, and 3 , respectively.

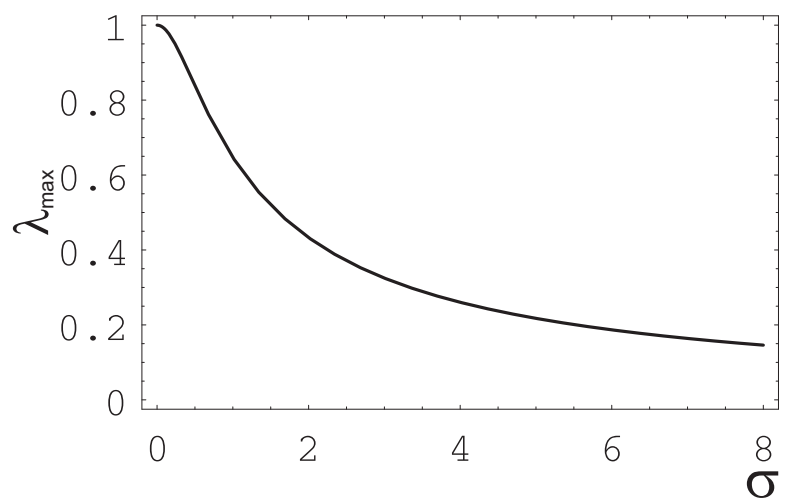

Figure 3. Dependence of the MI gain maximum $\lambda_{\max }$ on the nonlocality parameter $\sigma$ for the instability regime $(\mathrm{g})$.

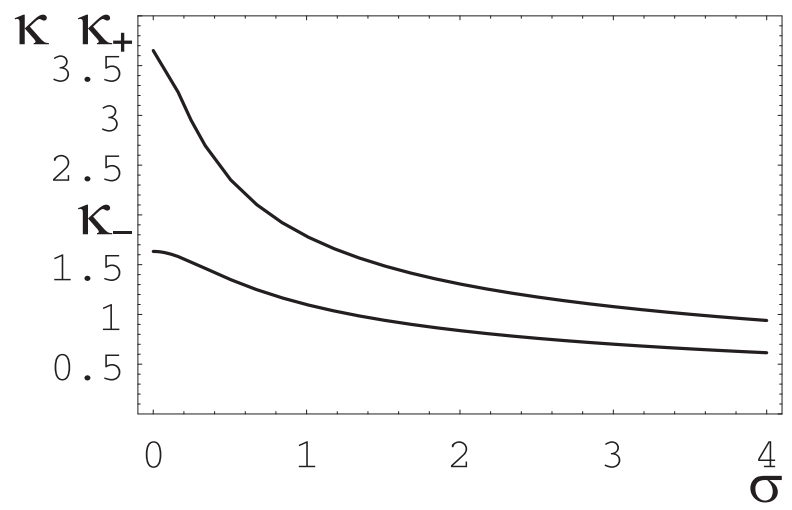

Figure 4. Dependence of limiting values $\kappa_{ \pm}$of modulation wave numbers on the nonlocality parameter $\sigma$ for the instability regime (e). The instability bandwidth shrinks with increasing nonlocality. 


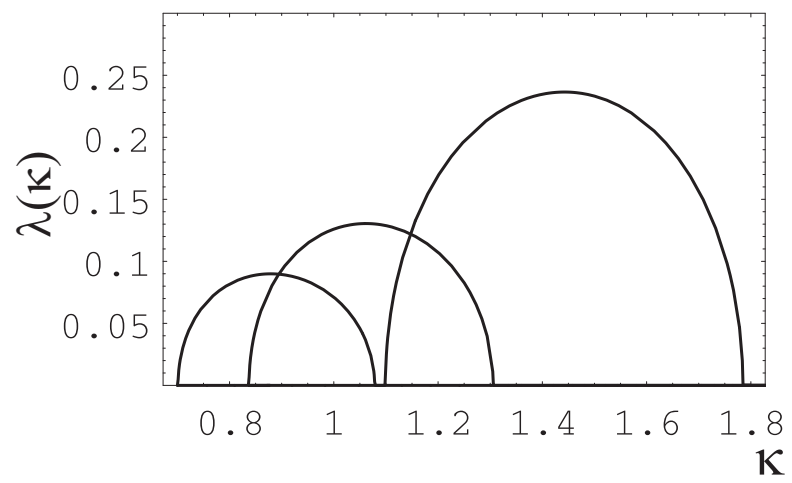

Figure 5. MI gain spectra for the instability regime (e) for different values of nonlocality parameter $\sigma$. Three curves correspond (from right to left) to $\sigma=1,2$, 3.

will follow [32, 17] and give a geometrical description of the nonlocality management in the case of the group-velocity mismatch.

Let us write the dispersion relation (66) in the form

$$
\left(\Omega-V_{1} \kappa\right)^{2}-\Omega_{1}^{2}=\frac{\Omega_{12}^{4}}{\left(\Omega-V_{2} \kappa\right)^{2}-\Omega_{2}^{2}} .
$$

This formula describes a mutual arrangement of two curves - a parabola on the left and a more complicated 'right' curve. We know from the previous exposition that the stability regime is provided by four real solutions of the dispersion equation (6), or, in other words, by four intersection points of the above curves. If the number of intersection points is less than four, the system is unstable. We show below that nonlocality of a medium provides an efficient tool to manage stability/instability properties giving a possibility to assure stability of a system which is unstable in a local medium.

Figure 6 corresponds to light wave propagation in a local medium $(\sigma=0)$ with the parameters $\mu_{1}=\mu_{2}=1, u_{0}=v_{0}=1, d_{1}^{2}=d_{2}^{2}=4, \alpha=2 / 3, \kappa^{2}=2$, and $V_{1}=V_{2}=0$, i.e., zero group-velocity mismatch. The choice of parameters gives $\Omega_{1}^{2}=\Omega_{2}^{2}=2$ and $\Omega_{12}^{4}=16 / 9$ and means individual stability of both waves. There are four real intersection points of the parabola with the 'right' curve, the latter consists of two hyperbolae and a curve in the lower half plane with two singular points. Therefore, this situation corresponds to absolute stability. Let us now introduce the velocity mismatch, taking $V_{1}=0$ and $V_{2}=\sqrt{2}$. The 'right' curve shifts horizontally, losing two intersection points and thereby producing instability (figure 7). If, however, nonlocality is coming into play [we take $\sigma=1$ and recalculate $\Omega_{1}^{2}, \Omega_{2}^{2}$ and $\Omega_{12}^{4}$ in accordance with (7)], the four intersection points occur again, and stability is restored (figure 8).

Our second example is concerned with a stable-unstable wave pair determined by the parameters $\mu_{1}=-1.5, \mu_{2}=1, u_{0}=v_{0}=0.6, \alpha=2 / 3, \kappa^{2}=1$ which correspond to $\Omega_{1}^{2}=1.1025, \Omega_{2}^{2}=-0.11$ and $\Omega_{12}^{4}=-0.0864$. For the group-velocity mismatch $\left(V_{1}=0.2, V_{2}=0\right)$ there are only two intersection points - the system is unstable (figure 9). Retaining this mismatch but allowing nonzero nonlocality $(\sigma=0.5)$, we restore the 


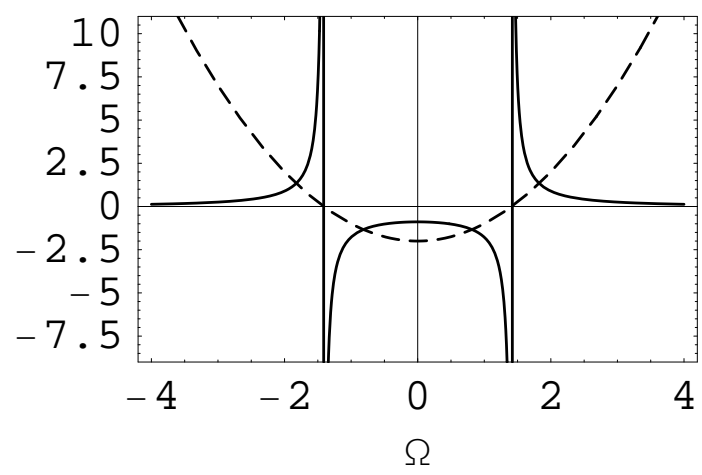

Figure 6. Zero group-velocity mismatch $\left(V_{1}=V_{2}\right)$ : four intersection points of the curves (the parabola is depicted by dashed line) defined in (13) provide stability of the continuous wave solution for a local medium $(\sigma=0)$ with the parameters $d_{1}^{2}=d_{2}^{2}=4$, $\mu_{1}=\mu_{2}=1, \kappa^{2}=2, u_{0}^{2}=v_{0}^{2}=1$.

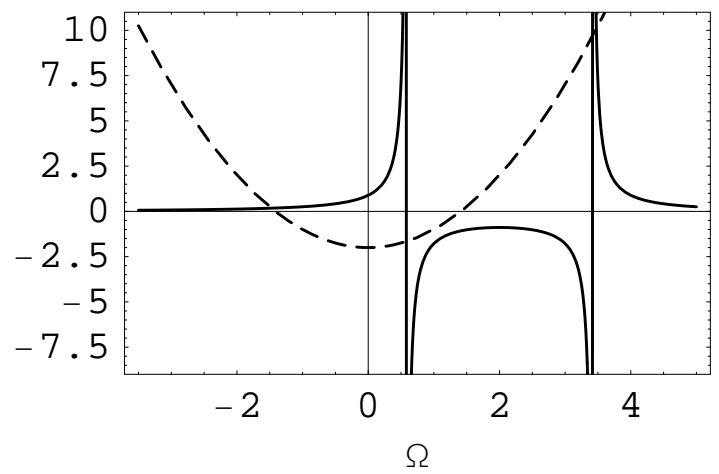

Figure 7. Breaking of stability (two intersection points only) in a local medium $(\sigma=0)$ caused by nonzero group-velocity mismatch $\left(V_{1}=0, V_{2}=\sqrt{2}\right)$. Other parameters are the same as in figure 6

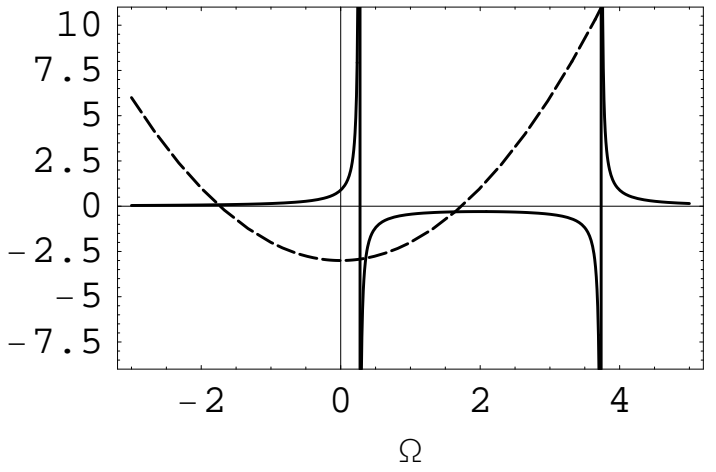

Figure 8. Restoration of stability (four intersection points) in a nonlocal medium $(\sigma=1)$ with nonzero group-velocity mismatch $\left(V_{1}=0, V_{2}=\sqrt{2}\right)$. Other parameters are the same as in figure 6 


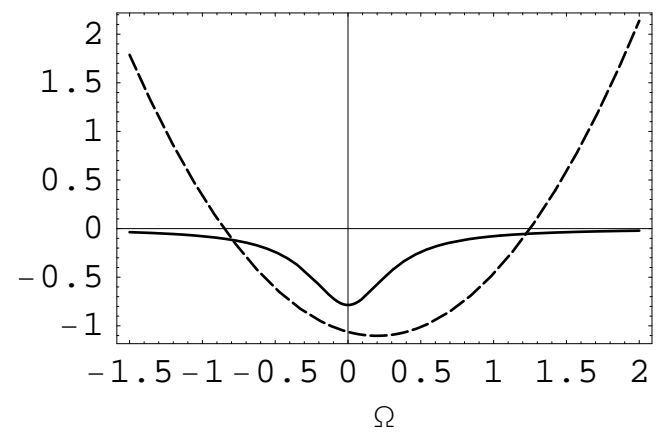

Figure 9. Stable-unstable wave pair in a local medium with the group-velocity mismatch. The parameters are $\mu_{1}=-1.5, \mu_{2}=1, u_{0}^{2}=v_{0}^{2}=0.6, \alpha=2 / 3, \kappa^{2}=1$, $V_{1}=0.2, V_{2}=0$. The system is unstable because there are only two intersection points of both curves.

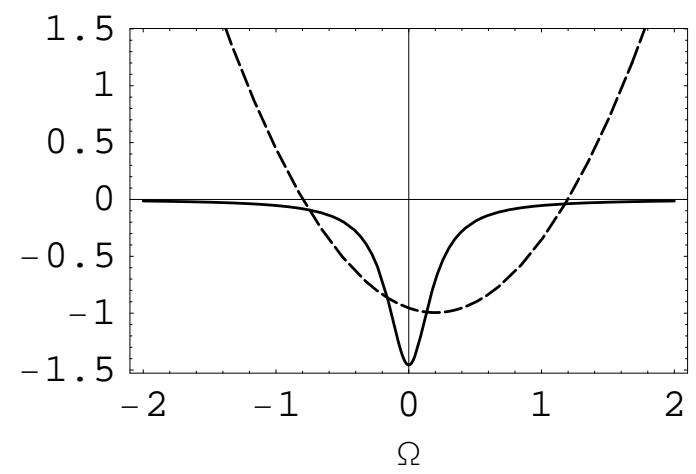

Figure 10. Restoration of stability of the stable-unstable wave pair in a nonlocal medium $(\sigma=0.5)$. Other parameters are the same as in figure 9 .

system stability (figure [10).

The last example provides the most emphatic illustration of the nonlocality management. Let us consider the unstable-unstable wave pair which corresponds to negative values of $\Omega_{1}^{2}$ and $\Omega_{2}^{2}$. It is stated in [17] that such a pair is always unstable in a local medium (see figure 11, no intersection points). The situation is drastically altered in a nonlocal medium. It is seen from (7) that $\Omega_{j}^{2}$ can change sign for sufficiently large nonlocality parameter $\sigma$, providing stability of the system. This possibility is exemplified by figure 12 .

\section{Conclusion}

We have investigated the MI of two interacting waves in a nonlocal Kerr medium, both for zero and nonzero group-velocity mismatch. The case of zero mismatch admits the full analytical description of stable/unstable regimes of the wave propagation. We have demonstrated that nonlocality suppresses the growth rate peak and bandwidth of instability. For the case of nonzero velocity mismatch we have revealed a crucial role of 


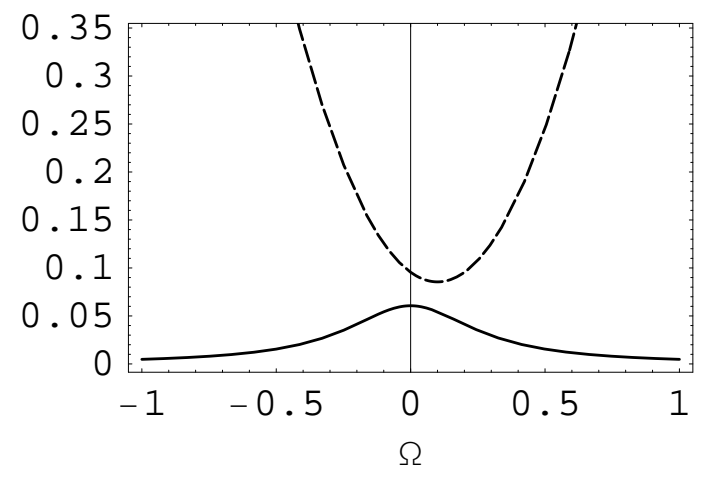

Figure 11. Unstable-unstable wave pair in a local medium. The parameters are $\mu_{1}=\mu_{2}=0.3, u_{0}=v_{0}=0.6, \alpha=2 / 3, \kappa^{2}=1, V_{1}=0.1, V_{2}=0$ which correspond to $\Omega_{1}^{2}=\Omega_{2}^{2}=-0.0855$. There are no intersection points, the system is absolutely unstable.

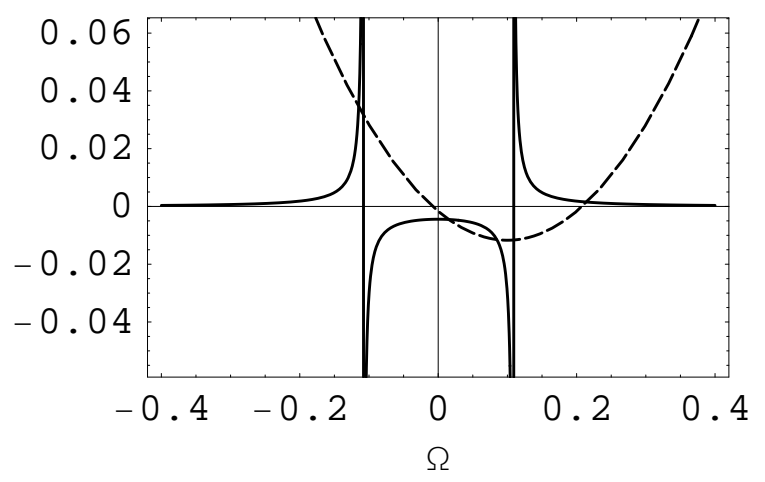

Figure 12. Unstable-unstable wave pair in a nonlocal medium $\left(\sigma=3, \Omega_{1}^{2}=\Omega_{2}^{2}=\right.$ 0.0117). Other parameters are the same as in figure 11. The system is stable at the cost of nonlocality management.

nonlocality in management of the stability properties in the system under consideration. In particular, we can provide stability of the nonlocal system in a regime when its local counterpart demonstrates absolute instability.

\section{References}

[1] Askar'yan G A 1962 Zh. Eksp. Teor. Fiz. 421576 [Sov. Phys.-JETP 15 1088]

[2] Taniuti T and Washimi H 1968 Phys. Rev. Lett. 21209

[3] Hasewaga A 1970 Phys. Rev. Lett. 241165

[4] Marklund M, Schukla P K, Stenflo L and Lundin J 2006 Phys. Scr. 74373

[5] Benjamin T B and Feir J E 1967 J. Fluid Mech 27417

[6] Bespalov V I and Talanov V I 1966 Zh. Eksp. Teor. Fiz. Pis'ma Red. 3471 [JETP Lett. 3 307]

[7] Ostrovskii L A 1966 Zh. Eksp. Teor. Fiz. 511189 [Sov. Phys.-JETP 24 797]

[8] Andersen D and Lisak M 1984 Opt. Lett. 9468

[9] Shukla P K and Rasmussen J J 1986 Opt. Lett. 11171

[10] Wu B and Niu Q 2001 Phys. Rev. A 64 061603(R) 
[11] Konotop V V and Salerno M 2001 Phys. Rev. A 65 021602(R)

[12] Hasewaga A 1984 Opt. Lett. 9288

[13] Abdullaev F K, Darmanyan S A and Garnier J 2002 Prog. Opt. 44303

[14] Berkhoer A L and Zakharov V E 1970 Zh. Eksp. Teor. Fiz. 58903 [Sov. Phys.-JETP 31486 ]

[15] Agrawal G P 1987 Phys. Rev. Lett. 59880

[16] Tanemura T and Kikuchi K 2003 J. Opt. Soc. Am. B 202502

[17] Kourakis I and Shuhla P K 2006 Eur. J. Phys. B 50321

[18] Litvak A G, Mironov V A, Fraiman G M and Yunakovskii A D 1975 Sov. J. Plasma Phys. 131

[19] Pecseli H L and Rasmussen J J 1980 Plasma Phys. 22421

[20] Peccianti M, Conti C, Alberici E and Assanto G 2004 Laser Phys. Lett. 225

[21] Kivshar Yu S and Peyrard M 1992 Phys. Rev. A 463198

[22] Perez-Garcia V M, Konotop V V and Garcia-Ripoll J J 2000 Phys. Rev. E 624300

[23] Doktorov E V and Molchan M A 2007 Phys. Rev. A 75053819

[24] Krolikovski W, Bang O, Nikolov N I, Neshev D, Wyller J, Rasmussen J J and Edmundson D 2004 J. Opt. B: Quantum Semiclass. Opt. S288

[25] Krolikowski W, McCarthy G, Saffman M, Bang O, Wyller J and Rasmussen J J 2006 Trends in Lasers and Electro-Optics Research ed W T Arkin (New York: Nova Science Publ.) p 265

[26] Xu Zhiyong, Kartashov Ya V and Torner L 2006 Phys. Rev. E 73 055601(R)

[27] Kartashov Ya V, Torner L, Vysloukh V A and Mihalache D 2006 Opt. Lett. 311483

[28] Mihalache D, Mazilu D, Lederer F, Crasovan L-C, Kartashov Ya V, Torner L, Malomed B A 2006 Phys. Rev. E $\mathbf{7 4} 066614$

[29] Mihalache D, Mazilu D, Lederer F, Malomed B A, Kartashov Ya V, Crasovan L-C, Torner L 2006 Phys. Rev. E 73 025601(R)

[30] Kivshar Yu S and Agrawal G P 2003 Optical Solitons: From Fibers to Photonic Crystals (San Diego: Academic)

[31] Wyller J 2001 Physica D 15790

[32] Das K P and Sihi S 1979 J. Plasma Phys. 21183 